\title{
Treatment of fenestrated vertebrobasilar junction ruptured giant aneurysm associated with two inlets and two outlets: A case report
}

\author{
Yu-Chun Lu ${ }^{1,2}$, Kha-Liang Lee ${ }^{1,2}$, Shun-Tai Yang ${ }^{1,2,3}$, Yueh-Hsun Lu ${ }^{4,5}$ and I-Chang Su ${ }^{1,2,3 *}$ \\ ${ }^{1}$ Department of Neurosurgery, Taipei Medical University-Shuang Ho Hospital, Ministry of Health and Welfare, Taipei Medical University, New Taipei City, \\ Taiwan \\ ${ }^{2}$ Taipei Neuroscience Institute, Taipei Medical University, Taipei, Taiwan \\ ${ }^{3}$ Department of Surgery, School of Medicine, College of Medicine, Taipei Medical University, Taipei, Taiwan \\ ${ }^{4}$ Department of Radiology, Taipei Medical University-Shuang Ho Hospital, Ministry of Health and Welfare, Taipei Medical University, New Taipei City, Taiwan \\ ${ }^{5}$ Department of Radiology, School of Medicine, College of Medicine, Taipei Medical University, Taipei, Taiwan
}

\begin{abstract}
Background: Basilar fenestration, as a result of the failed fusion of the paired fetal longitudinal neural arteries, is the most common intracranial arterial fenestration. When a vertebra-basilar junction (VBJ) aneurysm is observed, the presence of an associated basilar fenestration should be suspected. Due to the fact that the anatomy of VBJ aneurysms associated with basilar fenestration is commonly complex, a precise analysis of the morphology is mandatory to a successful treatment strategy.

Case Presentation: A 63-year-old woman had acute consciousness disturbance with Glasgow Coma Scale of 6 (E1V1M4). Computed tomography (CT) and CT angiography revealed a diffuse subarachnoid hemorrhage secondary to a ruptured giant aneurysm (maximal dome size: $26 \mathrm{~mm}$ ) at VBJ. Three-dimensional rotational catheter angiography with volume rendering further demonstrated the unusual morphology of the aneurysm, which extended from bilateral distal vertebral arteries (VA) to two limbs of an unfused lower basilar artery (BA) segment. Notably, each limbs of the unfused BA segment gave rise to an anterior- inferior cerebellar artery. The aneurysm was successfully managed by stent-assisted coiling.
\end{abstract}

Conclusion: The strategy preserved the whole vertebro-basilar artery and therefore cerebellar arteries. The patient made complete recovery without neurologic sequelae.

\section{Abbreviations}

BA: Basilar artery; VBJ: Vertebra-basilar junction; VAs: Vertebral arteries; CT: Computed tomography; MRI: Magnetic resonance imaging

\section{Background}

Basilar artery (BA) fenestration is a normal anatomic variant which involves duplication of part or all of the BA. It is thought to occur when the paired fetal longitudinal neural arteries, precursors of the BAs, fail to fuse $[1,2]$. While it is one of the most common forms of intracranial arterial fenestration, most basilar fenestration occurs in the lower half of the BA, immediately above the vertebra-basilar junction (VBJ) [3]. Some suggest that altered flow dynamics in the presence of fenestrations may promote the aneurysmal development, but the causative association has not been well-defined [4]. A case series comprised of six proximal BA fenestration aneurysms at VBJ has suggested endovascular approach to be safe and efficient while detailing the aneurysm morphology and its relation to the fenestration [5]. In present case, we showed a successful endovascular treatment on a ruptured giant $\mathrm{VBJ}$ aneurysm with previously unreported morphology of two-inlets from bilateral vertebral arteries (VAs) and two-outlets to two fenestrated limbs of BA. To the best of literature review, none has identified its classification or appropriate treatment to this rarer than rare entity. The study was approved by our institutional review board.

\section{Case presentation}

A 63-year-old female presented in the emergency department with severe vomiting and acute consciousness change (Hunt and Hess score of 3). Non-contrast computed tomography (CT) revealed Fisher grade 4 diffused subarachnoid hemorrhage and an associated hydrocephalus (Figure 1A). A subsequent CT angiography revealed an anteriorly directed $26 \mathrm{~mm}$ sized giant saccular aneurysm at the vertebrobasilar system (Figure 1B).

The patient was promptly intubated, and the acute hydrocephalus was managed with an external ventricular drainage. Cerebral

*Correspondence to: I-Chang $\mathrm{Su}$, Department of Surgery, School of Medicine, College of Medicine, Taipei Medical University, Taipei, Taiwan, Tel: 886-2-22490088-8120; Fax: 886-2-28285217; E-mail: ichangsu@gmail.com

Key words: Aarterial fenestration, endovascular coiling, intracranial aneurysm, vertebro-basilar junction

Received: May 27, 2021; Accepted: June 09, 2021; Published: June 14, 2021 


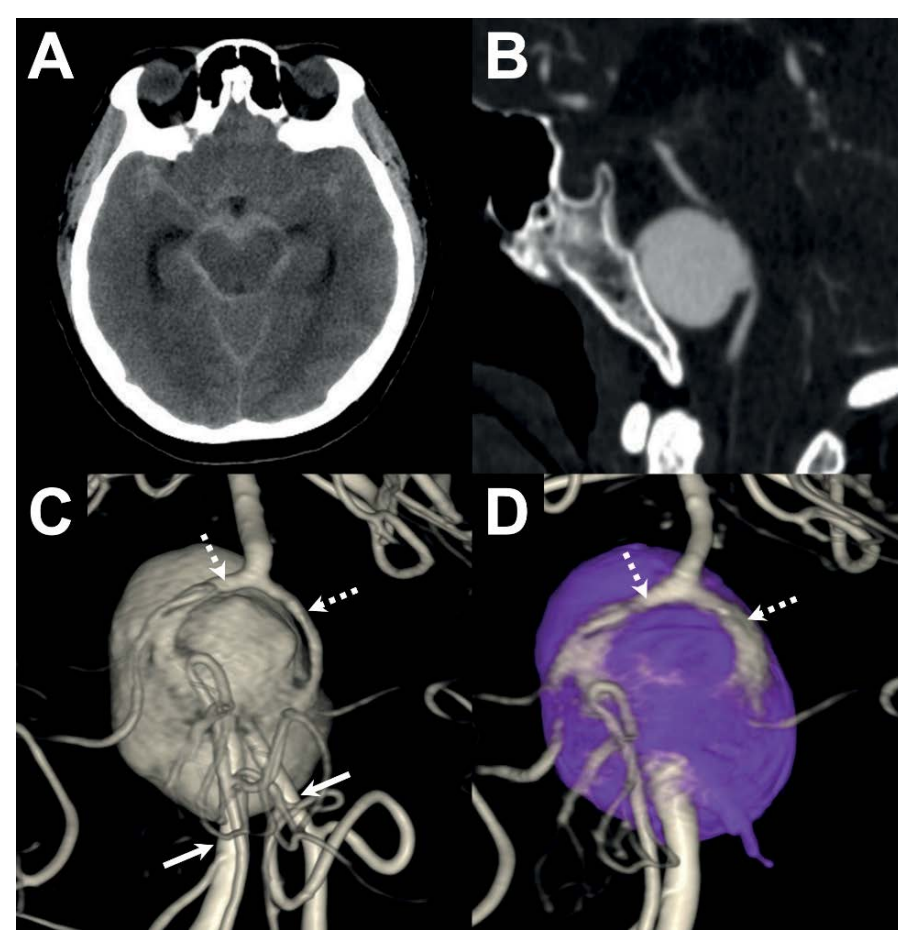

Figure 1. Extensive diffuse subarachnoid hemorrhage is seen over the basal cisterns and hydrocephalus in brain computed tomography (CT) (A). The sagittal view of the CT angiography shows an anterior directing giant saccular aneurysm at the vertebra-basilar system (B). Pre- (C) and post-treatment (D) posterior views of the three-dimensional rotational angiography with volume rendering were obtained from the left vertebral artery (VA) injection. Before treatment, the giant aneurysm is associated with two VA inlets (sold arrows) and two basilar fenestration outlet limbs (broken arrows) (C). Following endovascular treatment, the vertebra-basilar arterial system is reconstructed with a stent deployed from one side of the VA, across the aneurysm, toward the ipsilateral fenestration limb. The aneurysm is completely secured by dense aneurysm coil packing closing-off all possible inflow. Notice that the un-stented basilar fenestration limb is well opacified from the stented fenestration limb (broken arrows) (D)

angiogram was then performed to delineate the detailed aneurysm morphology. Volume rendering from the three-dimensional rotational angiography of the right VA injection revealed that the giant aneurysm was associated with two inlets and two outlets (Figure 1C). The two inlets were co-dominant in size and converged into the aneurysm pouch. The posterior inferior cerebellar artery arose from each inlet limb, indicating that the two inlets represented the VAs. The two outlets, which came out from the aneurysm dome and gave off anterior inferior cerebellar artery from their proximal segments, joined distally into a single $\mathrm{BA}$, indicating that each outlet limb represented a fenestration limb of the unfused lower BA (Figure 1).

Based on this morphological analysis, the endovascular treatment strategy was to place a stent from one inlet limb to a corresponding outlet limb, coil the aneurysm from the second inlet limb, and perform continuous coiling until the exit to the second outlet limb was closed off and the aneurysm was completely secured. Treatment should preserve both basilar fenestration limbs because each limb may send perforators to the brainstem. Therefore, in this manner, the second outlet limb would be perfused by retrograde filling from the stented outlet limb, leaving the aneurysm unfilled.

Dual antiplatelet medications (300 $\mathrm{mg}$ aspirin and $300 \mathrm{mg}$ clopidogrel) were administered before the procedure. The endovascular procedure was performed through the bilateral vertebral routes after placing a 6-Fr Envoy ${ }^{\circ}$ guiding catheter (Codman Neurosurgery, Raynham, Massachusetts, USA) in each distal V2 segment. A Headway ${ }^{\text {tw }}$ 21 microcatheter (Microvention-Terumo, Tustin, California, USA) was advanced in the left VA, across the aneurysm, and finally reaching the left outlet fenestration limb. A 4.0 × $22 \mathrm{~mm} \mathrm{LVIS}^{\text {tw }}$ stent (MicroventionTerumo, Tustin, California, USA) was then back-loaded and deployed from the left basilar fenestration limb to the left VA. An Excelsior ${ }^{\mathrm{m}}$ SL-10 microcatheter (Stryker Neurovascular, Kalamazoo, MI, USA) was then advanced in the right VA and placed within the aneurysm dome. A total of 49 Target $^{\circ}$ coils (Stryker Neurovascular, Kalamazoo, MI, USA) were deployed to secure the aneurysm. A final angiogram showed complete occlusion of the aneurysm, while both fenestration limbs and all cerebellar arteries remained patent. (Figure 1D) There were no procedure-related complications. No ischemic events were observed in post-procedural brain magnetic resonance imaging (MRI). The patient achieved good outcomes, with a modified Rankin scale of 0 at 3 months. A control angiogram at the 6-month follow-up revealed no aneurysm recanalization.

\section{Discussion and Conclusions}

This case revealed a ruptured giant aneurysm arose from the proximal bifurcation point of the VBJ fenestration, which led to an unusual two-inlet-two-outlet aneurysm geometry. The aneurysm was successfully treated with a good technical and clinical outcome after a precise analysis of the aneurysm morphology.

As with other intracranial arterial fenestrations, this anatomical variant is thought to be prone to aneurysm formation,[3] which is possibly related to a focal weakness within the vessel wall of the arterial fenestration and/or the increased hemodynamic turbulence at the proximal fenestration bifurcation [6,7]. This can result in aneurysms on the proximal aspect of the fenestration or on one of the fenestration limbs [8]. Moreover, the aneurysm can be single-lobed, bilobed, or even trilobed in morphology $[2,4]$. In other words, in the presence of an aneurysm on the VBJ, an associated basilar fenestration should be suspected, and aneurysms located at this specific location are commonly associated with complex anatomy.

We hypothesize that with two pre-existing basilar fenestration limbs above a normal VBJ, the aneurysm originally arose from the proximal bifurcation point of the VBJ fenestration (Figure 2A). As the aneurysm enlarged, it incorporated both VAs proximally and both fenestration limbs distally, and finally transformed into a giant aneurysm with a two-inlet-two-outlet configuration (Figure 2B,2C).

Currently, endovascular coiling/stenting is the first-line treatment for VBJ aneurysms associated with basilar fenestration [2,9]. However, two basic principles should be addressed in planning an appropriate endovascular strategy for such aneurysms. First, both basilar fenestration
A

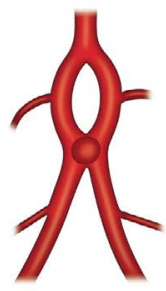

B

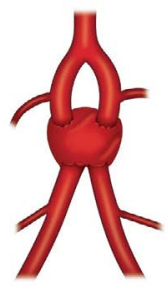

C

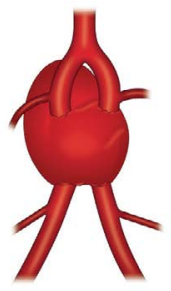

Figure 2. The proposed dynamic process of the aneurysm growth. Initially, the aneurysm originated from the progressively (B), it incorporated the proximal vertebral arteries and distal basilar fenestration limbs, proximal bifurcation point of the vertebra-basilar junction fenestration (A). As the aneurysm enlarged results in a giant aneurysm with two inlets and two outlets (C) 
limbs may be equal in size and one side may be dominant, but each limb may yield important perforating arteries to the brainstem [10]. Thus, it is desirable to preserve both limbs, if possible, regardless of the fenestration size. Second, in order to decrease the risk of recurrence, the aneurysm is better treated by both dense coil packing of the aneurysm dome and reducing the local hemodynamic turbulence around the VBJ complex [9]. In this case, with the advantage of having two inlets into the aneurysm, we first chose a stent with a higher metal coverage to reconstruct the vertebrobasilar arterial flow from one VA inlet to one basilar fenestration limb outlet. We then performed aneurysm coiling from the other VA inlet to densely pack the aneurysm and close off the aneurysm exit to the other basilar fenestration limb. Finally, the VA inlet used for coiling was also occluded with coils. In this manner, the vertebrobasilar arterial system was reconstructed into a better hemodynamic situation, and all possible inlets into the aneurysm were disrupted. More importantly, the un-stented basilar fenestration limb was well perfused by the retrograde filling from the stented basilar fenestration limb. This case demonstrates that endovascular coiling/stenting is a safe and effective treatment of choice for VBJ fenestration aneurysms, as long as the treatment strategy is well planned based on a precise analysis of the complex aneurysm morphology.

\section{Acknowledgements}

No grants were used for the current study. The authors have no relationships with any entity interested in the subject matter of this manuscript.

\section{Declarations}

\section{Ethical approval}

The study was approved by expedited review process of the TMUJoint Institutional Review Board (TMU-JIRB No.: N202003003).

\section{Competing Interest}

The authors declared no potential conflicts of interest with respect to the research, authorship, and/or publication of this article.

\section{Author Contributions}

Y.-C.L. and K.-L.L. formulated the theme and outline of the case report. Y.-C.L. and K.-L.L. reviewed the literature, drafted and revised the manuscript. S.-T.Y. and Y.-H.L. reviewed the literature and revised the manuscript. I.-C.S. reviewed, revised, and finalized the manuscript. All authors have read and agreed to the published version of the manuscript.

\section{Funding}

This research received no external funding.

\section{Availability of data and materials}

All data analyzed during this study are included in this manuscript.

\section{References}

1. Giuffre R, Sherkat S (1999) The vertebral artery: developmental pathology. J Neurosurg Sci 43: 175-189. [Crossref]

2. Gupta V, Ahuja CK, Khandelwal N, Kumar A, Gupta SK (2013) Treatment of ruptured saccular aneurysms of the fenestrated vertebrobasilar junction with balloon remodeling technique. A short case series and review of the literature. Interv Neuroradiol 19: 289298. [Crossref]

3. Sogawa K, Kikuchi Y, O'Uchi T, Tanaka M, Inoue T (2013) Fenestrations of the basilar artery demonstrated on magnetic resonance angiograms: an analysis of 212 cases. Interv Neuroradiol 19: 461-465. [Crossref]

4. Tasker AD, Byrne JV (1997) Basilar artery fenestration in association with aneurysms of the posterior cerebral circulation. Neuroradiology 39: 185-189. [Crossref]

5. Trivelato FP, Abud DG, Nakiri GS, de Castro Afonso LH, Ulhoa AC, et al (2016) Basilar Artery Fenestration Aneurysms: Endovascular Treatment Strategies Based on 3D Morphology. Clin Neuroradiol 26: 73-79. [Crossref]

6. Black SP, Ansbacher LE (1984) Saccular aneurysm associated with segmenta duplication of the basilar artery. A morphological study. J Neurosurg 61: 1005-1008. [Crossref]

7. Tsuei YS, Matsumoto Y, Ohta M, Nakayama T, Ezura M, et al (2009) Vertebrobasilar junction fenestration with dumbbell-shaped aneurysms formation: computational fluid dynamics analysis. Surg Neurol 72: S11-S19. [Crossref]

8. van Rooij SB, van Rooij WJ, Sluzewski M, Sprengers ME (2009) Fenestrations of intracranial arteries detected with 3D rotational angiography. AJNR Am J Neuroradiol 30: 1347-1350. [Crossref]

9. Zhu DY, Fang YB, Wu YN, Li Q, Duan GL, et al (2016) Treatment of fenestrated vertebrobasilar junction-related aneurysms with endovascular techniques. $J$ Clin Neurosci 28: 112-116. [Crossref]

10. Lasjaunias P, Berenstein A, Brugge Kt (2001) Functional anatomy of craniofacial arteries. Surgical neuroangiography 1: 280.

Copyright: $@ 2021$ I-Chang Su. This is an open-access article distributed under the terms of the Creative Commons Attribution License, which permits unrestricted use, distribution, and reproduction in any medium, provided the original author and source are credited. 